\title{
Influence of drying conditions on process properties and parameter identification for continuous fluidized bed spray agglomeration
}

\author{
Strenzke, G. ${ }^{a^{*}}$; Golovin, I. ${ }^{\text {; }}$ Wegner, M. ${ }^{\text { }}$; Palis, S. ${ }^{\text {be }}$; Bück, A.d ${\text {; Kienle, A. }{ }^{\text {b,c, }} \text { Tsotsas, E. }}^{\text {a }}$ \\ a Chair of Thermal Process Engineering, Institute of Process Engineering, Otto von Guericke \\ University, Magdeburg, Germany. \\ ${ }^{\mathrm{b}}$ Institute of Automation Engineering, Otto von Guericke University, Magdeburg, Germany.

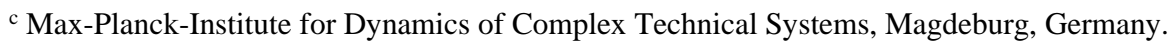 \\ ${ }^{\mathrm{d}}$ Institute of Particle Technology, Friedrich-Alexander University Erlangen-Nuremberg, Erlangen, \\ Germany. \\ e National Research University “Moscow Power Engineering Institute”, Moscow, Russia. \\ *corresponding author, Tel.: +49 39167 18320, E-Mail: gerd.strenzke@ovgu.de
}

\begin{abstract}
Agglomeration is a particle formulation process in which at least two primary particles are combined to form a new one. The growth of agglomerates depends on interactions of particles covered with wet spots that generated by depositions of binder droplets. This work experimentally compares the influence of external feed rate and sprayed binder content on product properties and process stability with internal separation at different drying conditions. Due to the identification of parameters a populations balance model (PBM) is developed. The PBM includes the agglomeration kernel function, which characterizes the kinetics, i.e. the rate at which primary particles build agglomerates.
\end{abstract}

Keywords: spray fluidized bed agglomeration; drying; continuous process; internal separation; population balances 


\section{Introduction}

Agglomeration is a particle formulation process in which at least two primary particles are combined to form a new one. The principle of fluidized bed spray agglomeration is especially used in chemical, food and pharmaceutical industry ${ }^{[1]}$. The advantages are good mixing and uniform high heat and mass transfer rates between particle, liquid and gas phase. Compared to previous batch processes the additional benefits of continuous processing are a constant product quality and higher throughputs. In case of fluidized bed spray agglomeration, a solidcontained liquid, called binder, is sprayed on the particles. The binder can be a solution, suspension or melt. The present work experimentally compares the influence of external feed rate and sprayed binder content on product properties and process stability with internal separation at different drying conditions. Product properties like particle size distribution or structure of agglomerates depend on these conditions and due to this, there are changes in product qualities, e.g. solubility, flowability or creation of dust free atmospheres. The growth of agglomerates depends on interactions of particles covered with wet spots that generated by depositions of binder droplets. Due to evaporation of water from the binder, wet connections between primary particles are transformed to solid bridges ${ }^{[2]}$. Therefore, the drying rate associated with gas temperature and moisture content finally determines the rate and quality of agglomeration.

\section{Materials and Method}

The starting materials for the fluidized bed and continuous feeding during the process are glass beads with distributed shapes, shown in Fig. 1. The Sauter diameter is $200 \mu \mathrm{m}$, measured optical by Camsizer, Retsch Technologies GmbH, Germany.
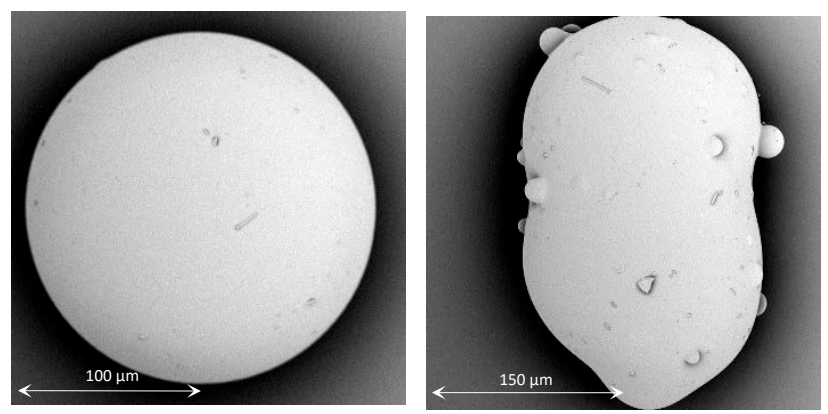

Fig. 1: different shapes of glass beads as primary particles

The operating binder is a solution of water and hydroxylpropylmethylcellulose (HPMC). HPMC is a biological binder also known as Pharmacoat and is used in food and pharmaceutical industries. In the present work it is used as a solution with water and a mass concentration of $4 \%$. To carry out the experiments a cylindrical fluidized bed is used, illustrated in Fig. 2. The fluidized bed is in a pilot plant scale. 


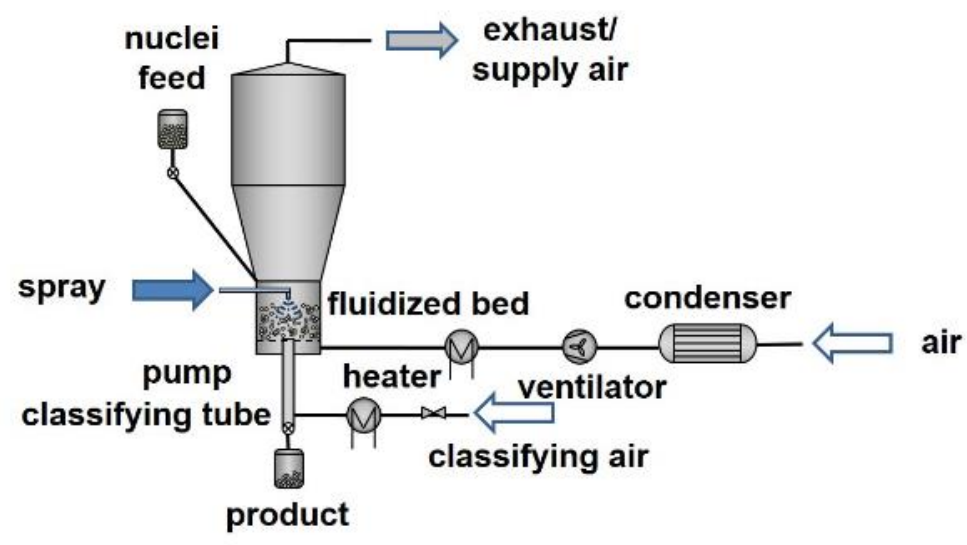

Fig. 2: Scheme of the fluidized bed

The particles are fluidized by heated ambient air air, which enters the fluidized bed chamber from the bottom throught a distributor plate. The Chamber of the fluidized bed has a diameter of $300 \mathrm{~mm}$. The spray nozzle, which atomized and sprayed the solution in top-spray configuration, is installed at a distance of $420 \mathrm{~mm}$ above the distributor plate of the fluidized bed. An external pump supply the feeding of the solution. Particles having the target size are continuously discharged by internal classification through a tube, which is centrally installed at the bottom of the fluidized bed. The important parameters (inlet temperature $-T_{\text {inlet }}$, air mass flow $-\dot{M}_{\text {inlet }}$, feed rate $-\dot{M}_{\text {Feed }}$, HPMC content - w and binder spray rate $-\dot{M}_{\text {Spray }}$ ) are shown in Table 1, all experiments started with a bed mass of $8 \mathrm{~kg}$.

Table 1: Experimental parameter

\begin{tabular}{llllll}
\hline Experiment & $\begin{array}{c}T_{\text {inlet }}, \\
{\left[{ }^{\circ} \mathrm{C}\right]}\end{array}$ & $\begin{array}{c}\dot{M}_{\text {inlet }}, \\
{[\mathrm{kg} / \mathrm{h}]}\end{array}$ & $\begin{array}{c}\dot{M}_{\text {Feed }}, \\
{[\mathrm{g} / \mathrm{min}]}\end{array}$ & $\begin{array}{c}\mathrm{W}, \\
{[\% \mathrm{HPMC}]}\end{array}$ & $\begin{array}{c}\dot{M}_{\text {Spray }}, \\
{[\mathrm{g} / \mathrm{min}]}\end{array}$ \\
\hline T1F150 & 80 & 275 & 150 & 4 & 55.5 \\
T1F250 & 80 & 275 & 250 & 4 & 55.5 \\
T1F350 & 80 & 275 & 350 & 4 & 55.5 \\
T2F150 & 100 & 275 & 150 & 4 & 55.5 \\
T2F250 & 100 & 275 & 250 & 4 & 55.5 \\
T2F350 & 100 & 275 & 350 & 4 & 55.5 \\
\hline
\end{tabular}

\section{Results and Discussion}

The Comparisons of the results of the 4 Experiments, shown in Fig. 3 and Fig. 4, are indicate that the product size of the experiments at $100{ }^{\circ} \mathrm{C}$ are smaller and the product size increase with decreasing feed rate. A break takes place during the experiment at $80{ }^{\circ} \mathrm{C}$, for a feed rate of $150 \mathrm{~g} / \mathrm{min}$ (at $35 \mathrm{~min}$ ). 

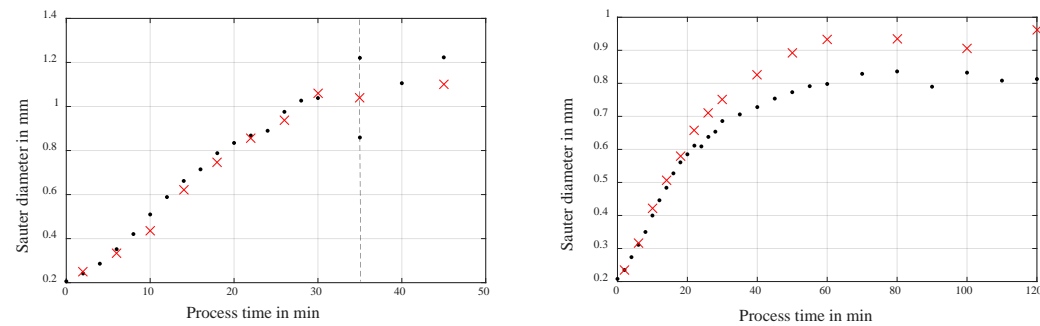

Fig. 3: Sauter diameter of bed (·) and product (x) samples for $150 \mathrm{~g} / \mathrm{min}$ at $80{ }^{\circ} \mathrm{C}(\mathrm{left})$ and $100{ }^{\circ} \mathrm{C}$ (right)
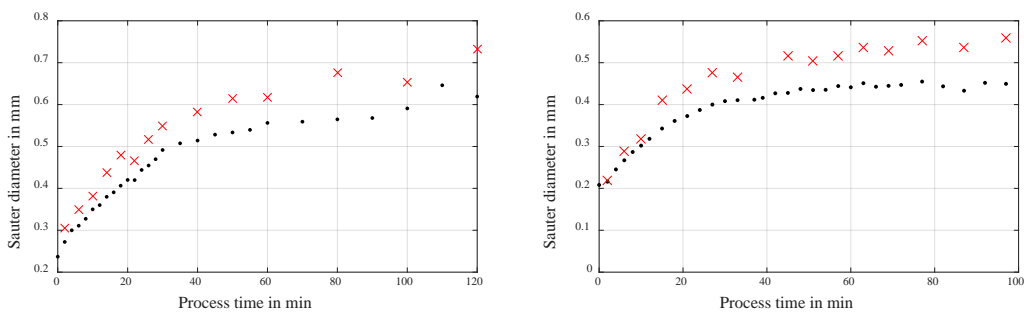

Fig. 4: Sauter diameter of bed (·) and product (x) samples for $350 \mathrm{~g} / \mathrm{min}$ at $80{ }^{\circ} \mathrm{C}(\mathrm{left})$ and $100{ }^{\circ} \mathrm{C}$ (right)

The reason is the lower evaporation rate of the water content from the solid bridges between the glass beads at lower temperature. Due to this, the residence time of with binder wetted surface get longer. It is more time to collide and connect with other wetted surfaces. The influence of the feed rate is also clear visuable, especially for the experiments at $100{ }^{\circ} \mathrm{C}$. Here at low feed rate the product particles reached a size of $0.9 \mathrm{~mm}$. With increasing the feed rate the size is getting smaller. The structure analysis of the agglomerates with the Scatter electrone microscope (SEM) prove the study, that at lower temperatures more binder is on the particles. The structure analysis of the agglomerates are shown in Fig. 5 and Fig. 6. For both temperatures the lowest feed rate indicates a partial coating. This means a part up to the whole particle surface is covered with the binder. With increasing the feed rate, more particles enter the spray zone and the binder solution is more distributed over the bed particles. The particles in figure 6 are indicates optically a more compact structure as in figure 5 , which is proved by Camsizer measurements, shown in Table 2. This indicates that the dense of the agglomerates increase by the inlet temperature, which is confirmed by Dadkhah ${ }^{[3]}$.

Table 2: Density of agglomerates at experimental parameters

\begin{tabular}{cccc}
\hline Temperature $\left[{ }^{\circ} \mathrm{C}\right]$ & F150 & F250 & F350 \\
\hline 80 & $>1.2$ & 1.44 & 1.6 \\
100 & 1.47 & 1.7 & 1.7 \\
\hline
\end{tabular}



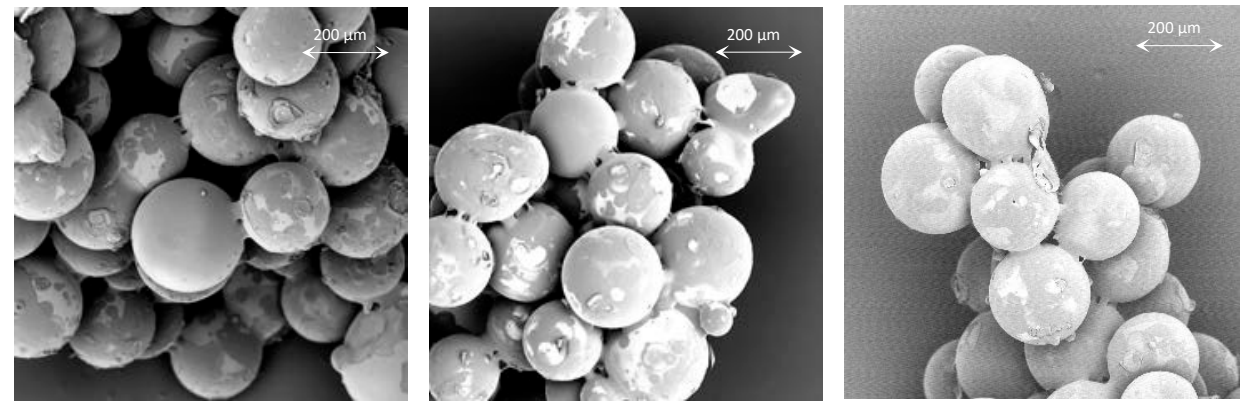

Fig. 5: SEM pictures of product particles at $80^{\circ} \mathrm{C}$ with $150 \mathrm{~g} / \mathrm{min}$ (left), $250 \mathrm{~g} / \mathrm{min}$ (middle) and $350 \mathrm{~g} / \mathrm{min}$ (right)
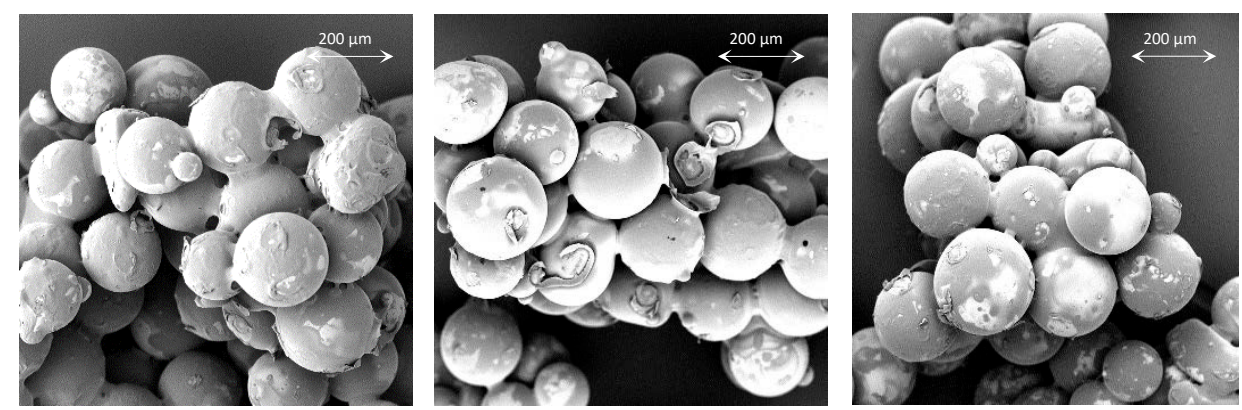

Fig. 6: SEM pictures of product particles at $100{ }^{\circ} \mathrm{C}$ with $150 \mathrm{~g} / \mathrm{min}$ (left), $250 \mathrm{~g} / \mathrm{min}$ (middle) and $350 \mathrm{~g} / \mathrm{min}$ (right)

In order to describe the dynamic behaviour of continuous fluidized bed spray agglomeration, the following population balance model can be used:

$$
\frac{\partial n(t, v)}{\partial t}=\dot{n}_{f e e d}(t, v)-\dot{n}_{\text {prod }}(t, v)+\dot{n}_{a g g}(t, v),
$$

where $n$ symbolizes the number density distribution, $v$ denotes the particle volume, $\dot{n}_{f e e d}(t, v), \dot{n}_{\text {prod }}(t, v)$ and $\dot{n}_{a g g}(t, v)$ represent particle fluxes due to the external particles feed, the reflux of the product particles and agglomeration process respectively. The formation and growth of agglomerates including initial condition can be described by:

$$
\begin{gathered}
\dot{n}_{a g g}(t, v)=\frac{1}{2} \int_{0}^{v} \beta(t, u, v-u) n(t, u) n(t, v-u) d u-\int_{0}^{\infty} \beta(t, u, v) n(t, v) n(t, u) d u,(2) \\
n(t=0, v)=n_{0}(v),
\end{gathered}
$$

where $\beta(t, u, v)$ is an agglomeration function (kernel) characterizing the kinetics of the agglomeration process. This kernel can be represented as a product of a coalescence kernel 
$\beta(u, v)$, which depends only on the volume of the agglomerating particles and an agglomeration efficiency $\beta_{0}(t)$, which is a positive possibly time-dependent function:

$$
\beta(t, u, v)=\beta_{0}(t) \beta(u, v) .
$$

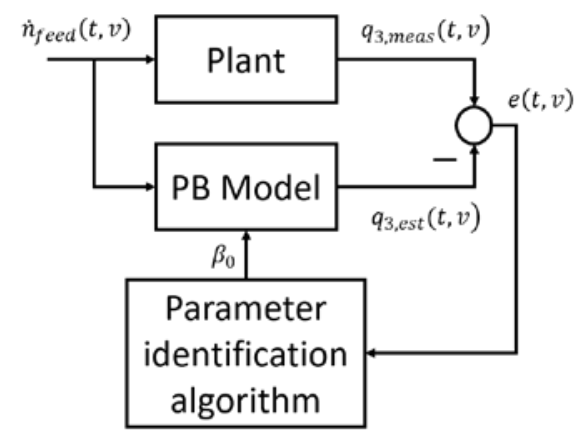

Fig. 7: Scheme of parameter identification

The Estimation of kinetics for an agglomeration is particularly challenging due to its highly nonlinear process dynamics. The coalescence kernel $\beta(u, v)$, being a nonnegative symmetric function of two variables, describes an impact of particle sizes on the probability of its effective collision. In order to understand the formation of agglomerates in a first step the mechanical kernel based on Brownian motion ${ }^{[7]}$ can be applied for simulation model:

$$
\beta(u, v)=\beta_{0}\left(v^{\frac{1}{3}}+u^{\frac{1}{3}}\right)\left(v^{-\frac{1}{3}}+u^{-\frac{1}{3}}\right)
$$

Assuming that time dependency of the agglomeration efficiency $\beta_{0}$ can be neglected, this scalar parameter can be estimated from the experimental particle size distributions using a parallel model approach Fig. $7^{[4]}$. Substituting this mechanical kernel into the population balance model a linear regression problem can be formulated:

$$
\min _{\beta_{0}} \int_{0}^{T}\left\|q_{3, \text { meas }}(t)-q_{3, \text { est }}\left(t, \beta_{0}\right)\right\|_{2}^{2} d t
$$

In order to compute the given population balance model the method of lines can be applied, where the spatial coordinate is discretized using the cell-average method ${ }^{[8]}$ on a logarithmic grid. The experimental and simulation results with Brownian motion kernel are depicted in Fig. 8. In Fig. 9 is shown a comparison of the initial and steady state distribution. It can be seen that using this theoretical kernel for continuous fluidized bed spray agglomeration 
process yields a qualitatively good approximation of the process particularly for steady-state region.
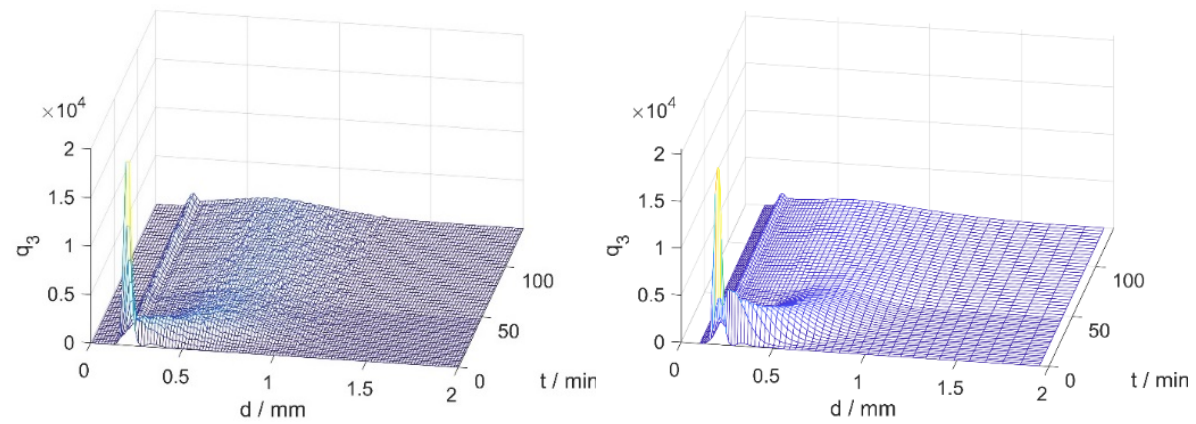

Fig. 8: Experimental (left) and simulated (right) particle size distribution $q_{3}$
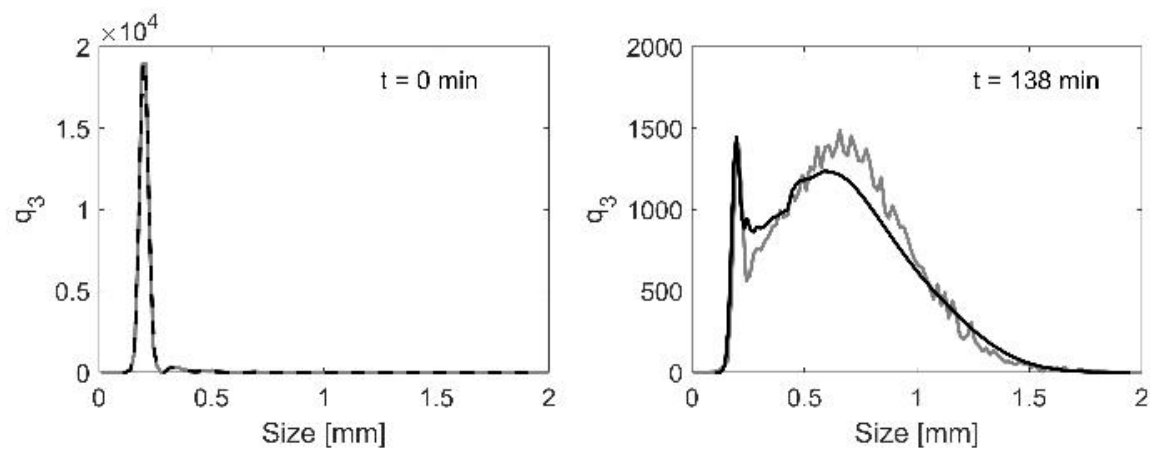

Fig. 9: Experimental (grey) and simulated (black) particle size distribution $q_{3}$ for the initial (left) and steady state (right) distribution

\section{Conclusions}

The Influence of the temperature and feed rate on the particle were investigated. The results of the experiments show that a lower temperature lead to bigger agglomerates. The increasing of the feed rate indicates smaller particles. A high temperature and high feed rate should be result in smaller product particles. The results of the structure investigations does not indicate a preferd shape, like sphericals or sticks, at different temperatures or feed rates.

The developed PBM model was qualitatively good approximation of the process particularly for steady-state region. In order to get more precise process approximation another kernel functions of more degrees of freedom, e.g. Laurent polynomials of two variables ${ }^{[6]}$, bilinear basis functions ${ }^{[5]}$, or time dependency of the agglomeration efficiency $\beta_{0}(t)$ can be taken into account. 
Influence of drying conditions on process properties and parameter identification for continuous fluidized bed spray agglomeration

\section{Acknowledgement}

This publication was supported by the Center of Dynamic Systems (CDS), funded by the EU-programme ERDF (European Regional Development Fund).

\section{References}

[1] Bück, A., Tsotsas, E., 2016. Encyclopedia of Food and Health. Vol.1. Oxford: Academic Press, Ch. Agglomeration, pp. $73-81$.

[2] Terrazas-Velarde, K., Peglow, M., Tsotsas, E., 2011, Kinetics of fluidized bed spray agglomeration for compact and porous particles, Chemical Engineering Science 66, pp. $1866-1878$

[3] Dadkhah, M., Tsotsas, E., 2014. Influence of process variables on internal particle structure in spray fluidized bed agglomeration

[4] Palis, S., Kienle, A., 2013. "Online parameter identification for continuous fluidized bed spray granulation", 5th International Conference on Population Balance Modelling PBM (Bangalore).

[5] Chakraborty, J., Kumar, J., Singh, M., Mahoney, A., Ramkrishna, D., 2015. Inverse problems in population balances. Determination of aggregation kernel by weighted residuals. Ind. Eng. Chem. Res. 54, pp. 10530-10538.

[6] Eisenschmidt, H., Soumaya, M., Bajcina, N., Le Borne, S., \& Sundmacher, K., 2017. Estimation of aggregation kernels based on Laurent polynomial approximation. Computers \& Chemical Engineering 103, pp. 210-217.

[7] Schwabl, F., 2006. Statistische Mechanik. Springer-Verlag Berlin Heidelberg.

[8] Kumar, J., 2006. Improved accuracy and convergence of Discretized Population Balance for Aggregation: The cell average technique. Chemical Engineering Science, 61, pp. 3327-3342. 